\title{
Shaping triangular picosecond laser pulses for electron photoinjectors
}

Mironov S.Yu. ${ }^{1}$, Poteomkin A.K. ${ }^{1}$, Kuzmin I.V., Gacheva E.I. ${ }^{1}$, Andrianov A.V. ${ }^{1}$, Zelenogorsky V.V. ${ }^{1}$, Krasilnikov M. ${ }^{2}$, Loisch G. ${ }^{2}$, Stephan F. ${ }^{2}$ and Khazanov E.A. ${ }^{1}$

\section{${ }^{1}$ Institute of Applied Physics of RAS, Nizhny Novgorod, Russia ${ }^{2}$ DESY, Zeuthen, Germany}




\section{Outline}

- Motivation for pulse shaping activity

- Theoretical model and results of numerical simulations

- Experimental results: achievements and drawbacks

- Conclusion 


\section{Motivation for laser pulse shaping at photoinjector}

- Active control of parameters of generated electron bunches: emittance, distribution of spatial charge: shape and duration;

- Development new methods for control of laser pulse intensity distribution at ps region;

- Improvement of laser pulse and beam quality;

- Development new methods for laser beam diagnostics;

Intermediate outcome of the activity is a formation of cylindrical and ellipsoidal laser pulses
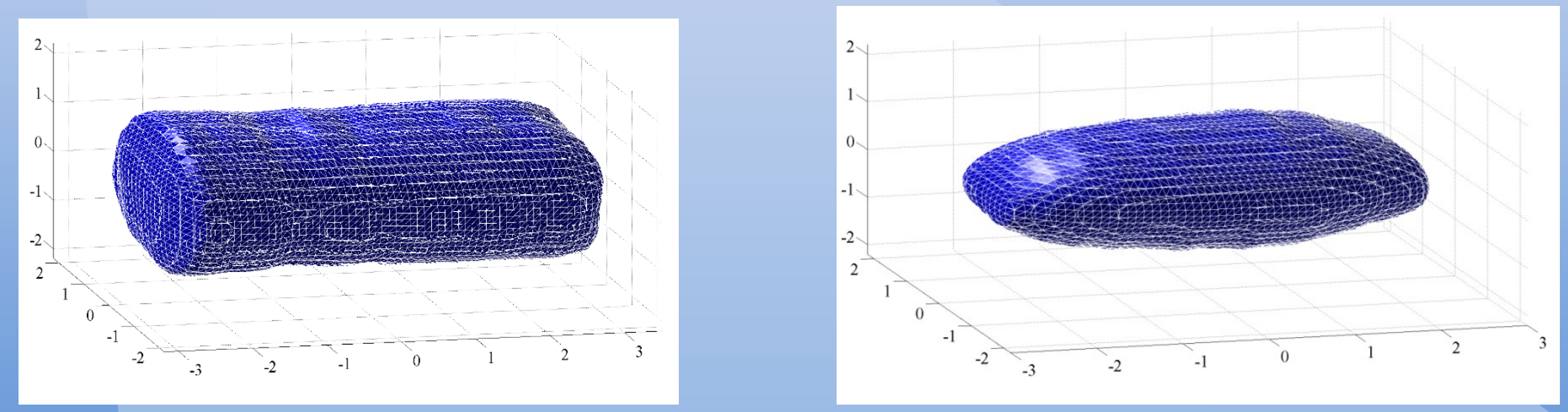


\section{Optimal spectrum cutting}
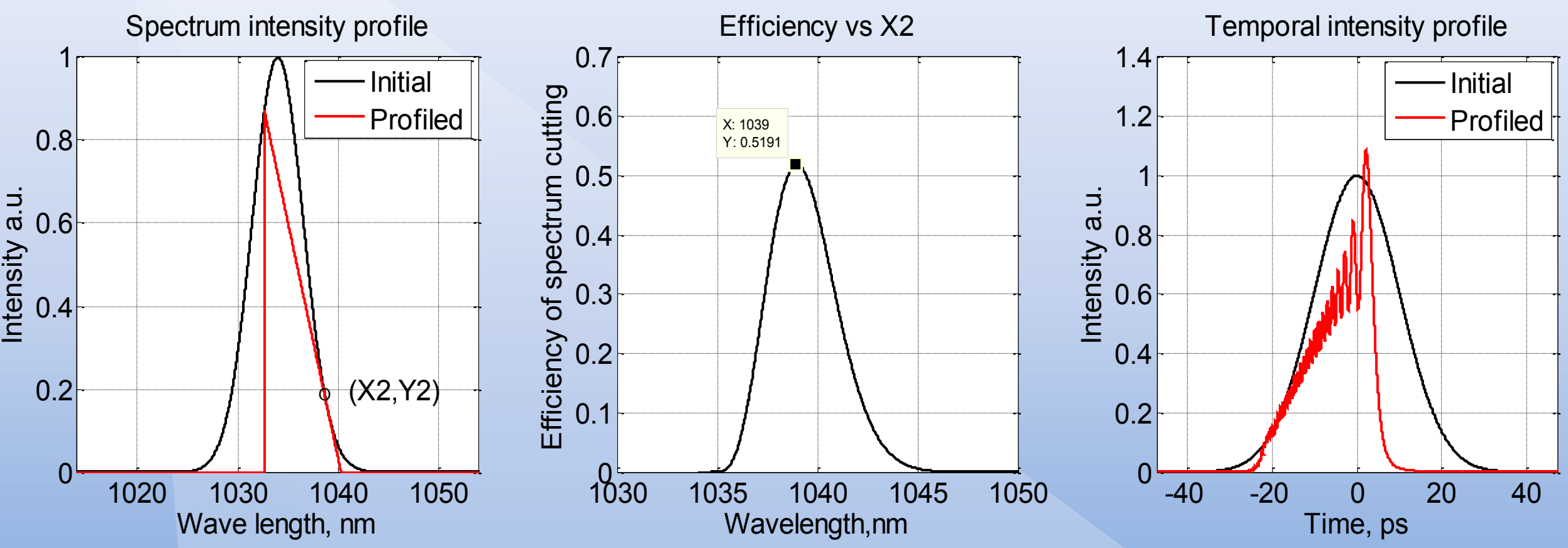

If $\mathrm{T} / \mathrm{TF} \sim 90$ The sharp spectral intensity distribution is a reason for oscillation on temporal shape

Optimal energy conversion is about $52 \%$

The smoothed spectral masks can be used for minimizing oscillations on the temporal shape 
AD Federal Research Center The Institute of Applied Physics of the Russian Academy of Sciences

\section{Experimental results}

\section{Spectral intensity distributions}

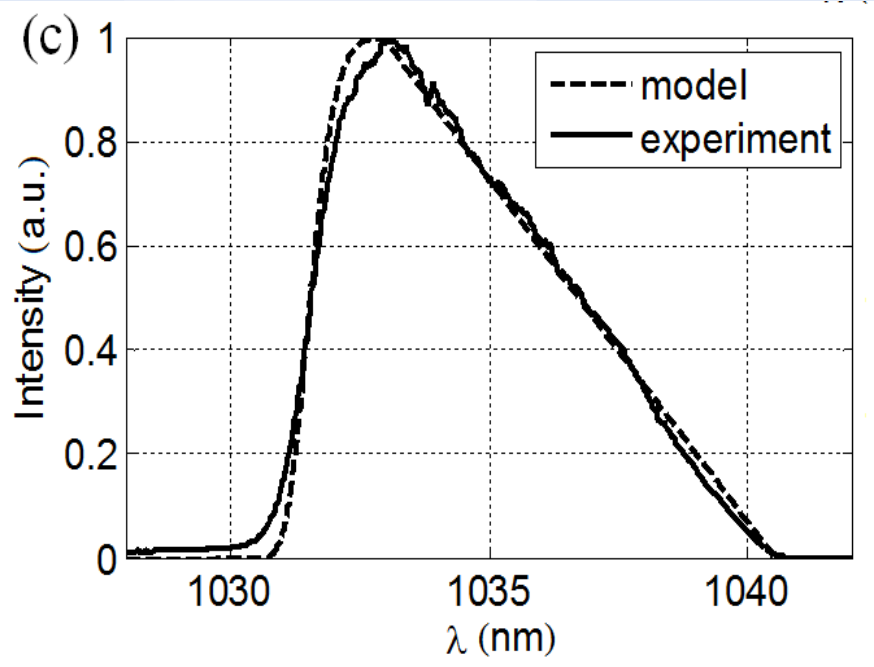

Temporal intensity distribution model

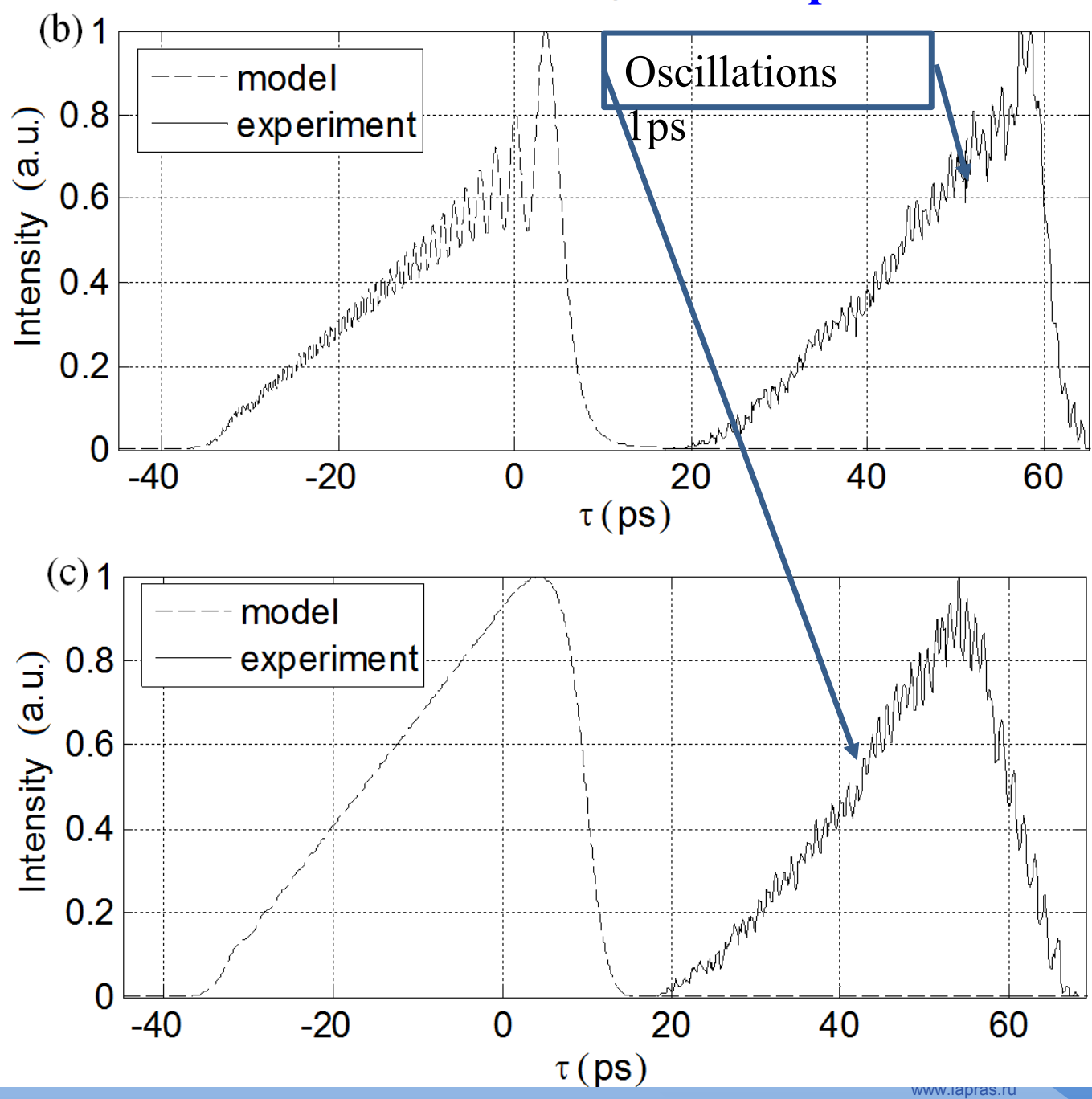




\section{Conclusion}

- We have demonstrated experimental implementation of picosecond chirped laser pulses with quasi-triangular intensity distribution in time.

- Shaping of quasi-triangular intensity distribution has been analyzed numerically, its basic principles and conditions for their optimal realization have been determined.

- We have revealed periodic distortions of the temporal intensity distribution profile at different experimental conditions associated with the SLM matrix. Such distortions are, evidently, caused by the molecular response of the NLCs used in the modulator to the laser radiation field. 


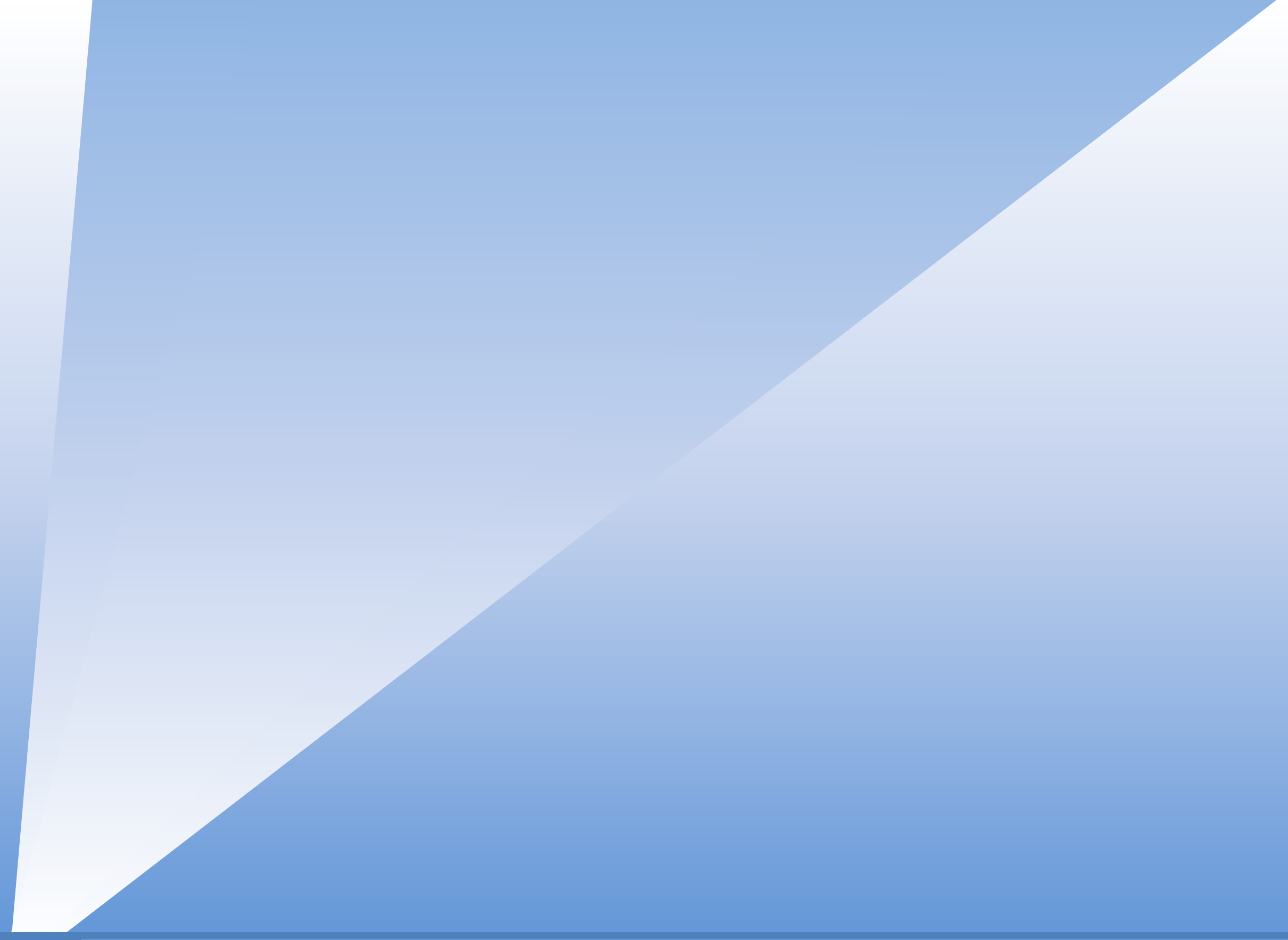




\section{Update of laser driver for electron photo injector at IAP RAS}

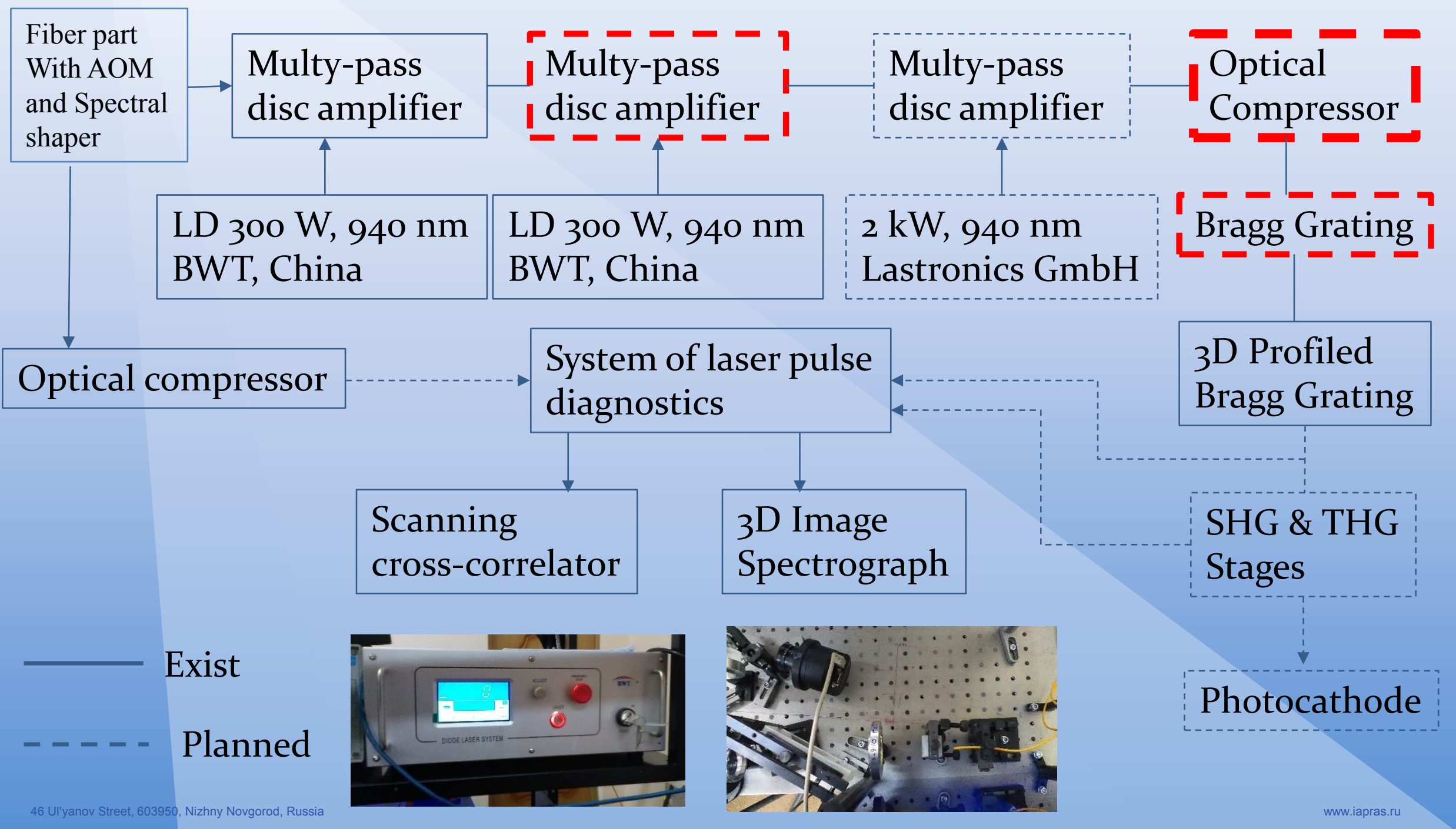

